\title{
Hnf4a deletion in the mouse kidney phenocopies Fanconi renotubular syndrome
}

\author{
Sierra S. Marable, ${ }^{1,2}$ Eunah Chung, ${ }^{1}$ Mike Adam, ${ }^{2}$ S. Steven Potter, ${ }^{2}$ and Joo-Seop Park ${ }^{1,2}$ \\ 'Division of Pediatric Urology and 'Division of Developmental Biology, Cincinnati Children's Hospital Medical Center \\ (CCHMC), Cincinnati, Ohio, USA.
}

Different nephron tubule segments perform distinct physiological functions, collectively acting as a blood filtration unit. Dysfunction of the proximal tubule segment can lead to Fanconi renotubular syndrome (FRTS), with major symptoms such as excess excretion of water, glucose, and phosphate in the urine. It has been shown that a mutation in HNF4A is associated with FRTS in humans and that $H n f 4 a$ is expressed specifically in proximal tubules in adult rat nephrons. However, little is known about the role of $\mathrm{Hnf4a}$ in nephrogenesis. Here, we found that $\mathrm{Hnf4a}$ is expressed in both presumptive and differentiated proximal tubules in the developing mouse kidney. We show that $\mathrm{Hnf4a}$ is required for the formation of differentiated proximal tubules but is dispensable for the formation of presumptive proximal tubules. Furthermore, we show that loss of Hnf4a decreased the expression of proximal tubule-specific genes. Adult Hnf4a mutant mice presented with FRTSlike symptoms, including polyuria, polydipsia, glycosuria, and phosphaturia. Analysis of the adult Hnf4a mutant kidney also showed proximal tubule dysgenesis and nephrocalcinosis. Our results demonstrate the critical role of $\mathrm{Hnf} 4 \mathrm{a}$ in proximal tubule development and provide mechanistic insight into the etiology of FRTS.

Conflict of interest: The authors have declared that no conflict of interest exists.

Submitted: September 14, 2017

Accepted: June 19, 2018

Published: July 25, 2018

Reference information: JCI Insight. 2018;3(14):e97497. https://doi.org/10.1172/jici. insight.97497.

\section{Introduction}

Fanconi renotubular syndrome (FRTS) is caused by proximal tubule dysfunction leading to impaired reabsorption of water and organic solutes, such as glucose, amino acids, and phosphate (1-3). The proximal tubule is responsible for approximately $65 \%$ of all reabsorption in the nephron, including $70 \%$ of water, $99 \%-100 \%$ of glucose, and $85 \%$ of phosphate (4-6). In FRTS, impaired reabsorption results in excessive excretion of these solutes and water in the urine. In recent years, it was shown that single gene mutations are associated with hereditary FRTS. In a subset of patients, a heterozygous mutation in the $H N F 4 A$ gene has been found (7). FRTS only affects the proximal tubule, not other segments of the nephron, implying that the function of $H N F 4 A$ is important specifically in the proximal tubule (5).

$H N F 4 A$ encodes a member of the nuclear receptor superfamily of ligand-dependent transcription factors (8) Hnf4a is at the center of a complex regulatory network that controls metabolism and hepatocyte differentiation $(9,10)$. However, its role in the kidney is poorly understood. An analysis of gene expression in the developing mouse kidney identified Hnf4a as a candidate anchor gene within the proximal tubule, suggesting that its expression is specific to this segment of the kidney (11). In the adult rat nephron, Hnf4a is expressed specifically in proximal tubules (12). A recent study has shown that Hnf4a regulates expression of drug transporters in the rat kidney (13), many of which are specifically expressed in the proximal tubules. These data suggest that Hnf4a plays an important role in the proximal tubule. Here, we examined the role of Hnf4a in proximal tubule development using a murine model of nephron-specific deletion of Hnfta. We found that Hnf $4 a$ is required for the formation of differentiated proximal tubules but is not necessary for the formation of presumptive proximal tubules. Due to the resulting paucity of proximal tubules, the Hnf4a mutant mice presented with FRTS-like symptoms.

\section{Results}

Hnfta is expressed in both presumptive proximal tubules and differentiated proximal tubules. In order to investigate the role of Hnf4a in the developing nephron, we examined its spatial and temporal expression during nephrogenesis. We performed lineage tracing of the nephron progenitors by employing Six2GFPcre, which 
A

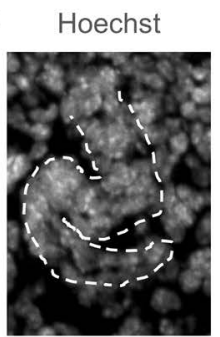

B

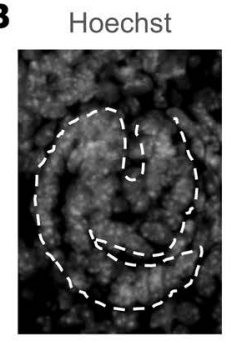

C Hoechst

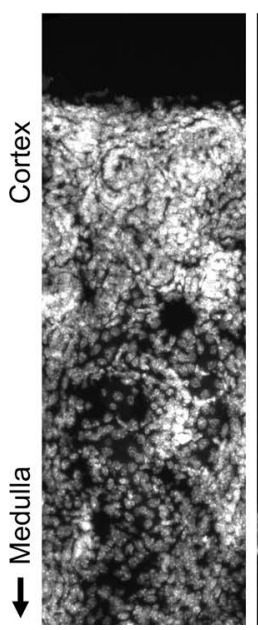

Jag1

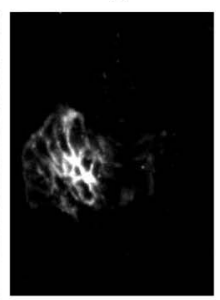

Jag1

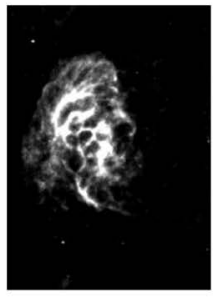

LTL

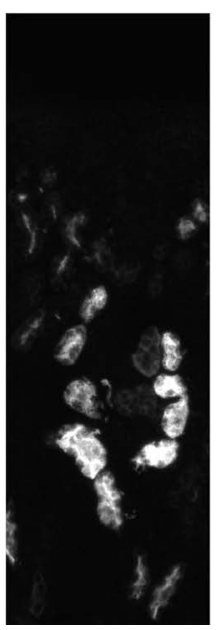

Hnf4a

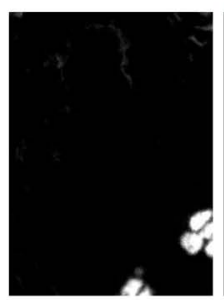

$\mathrm{Hnf4a}$

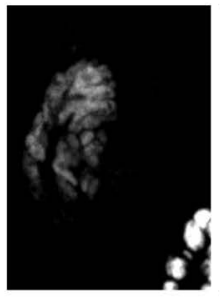

$\mathrm{Hnf} 4 \mathrm{a}$

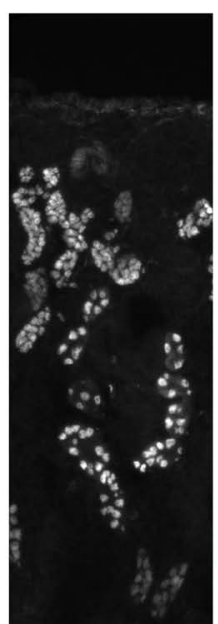

Jag1 Hnf4a

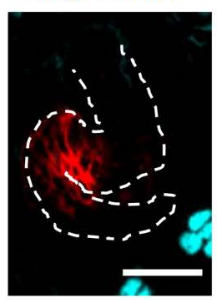

Jag1 Hnf4a

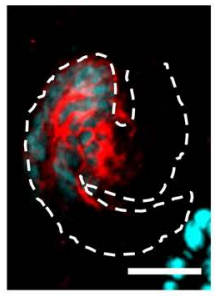

LTL Hnf4a

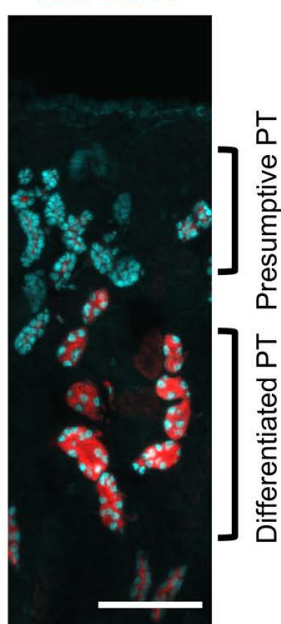

Figure 1. Hnf4a is expressed in the developing nephron. (A) In nascent S-shaped body (SSB), Hnf4a is not detected. Representative image of $n=3$. Scale bar: $25 \mu \mathrm{m}$. (B) $H n f 4 a$ is expressed in a subset of Jag1+ cells in the medial segment of the elongated SSB. Scale bar: $25 \mu \mathrm{m}$. Image is representative of $n=3$. (C) Hnf4a is detected in presumptive proximal tubule (PT) cells and differentiated PT cells. Presumptive PT cells show weak Lotus tetragonolobus lectin (LTL) staining and are located more cortically, where early stages of nephrogenesis occur. Differentiated PT cells show strong LTL staining and are located closer to the medullary region. Image is representative of $n=3$. Scale bar: $100 \mu \mathrm{m}$.

specifically targets mesenchymal nephron progenitors $(14,15)$. We found that, when cells in the nephron lineage were labeled with an enhanced yellow fluorescent protein (EYFP) reporter (16), all $\mathrm{Hnf}_{4} \mathrm{a}^{+}$cells were positive for EYFP (Supplemental Figure 1; supplemental material available online with this article; https://doi.org/10.1172/jci.insight.97497DS1), suggesting that Hnf4a is expressed only in the nephron lineage in the kidney. In the nascent S-shaped body, whose medial segment expresses Jag1, no Hnf4a was detected (Figure 1A). However, in the more mature and extended S-shaped body, where the Jag1 expression domain was expanded, Hnf4a was detected in a subset of Jag $1^{+}$cells (Figure 1B). It is likely that Hnf4a $\mathrm{a}^{+}$ cells in the extended S-shaped body develop into proximal tubules.

Lotus tetragonolobus lectin (LTL) staining is widely used to detect proximal tubule cells. LTL strongly binds to carbohydrates of glycoproteins, which are abundant in the proximal tubule cells $(17,18)$. We found that Hnf4a was expressed in LTL stain-positive proximal tubules (Figure 1C). With close inspection, we found 2 populations of LTL stain-positive cells (LTL-low and LTL-high) in the kidney (Figure 1C). LTLhigh cells showed strong LTL staining in their entire cell bodies. By contrast, LTL-low cells showed only weak LTL staining in their apical (luminal) membrane. Both LTL-high and LTL-low cells expressed Hnf4a. LTL-low cells are located more cortically where early nephrogenesis occurs, suggesting LTL-low cells are nascent proximal tubule cells. LTL-high cells are located closer to the medullary region, suggesting a more differentiated state. Furthermore, the distance between $\mathrm{Hnf}_{4} \mathrm{a}^{+}$nuclei was increased in LTL-high cells, suggesting that these cells were larger than LTL-low cells (Figure 1C). It is therefore likely that LTL-low and LTL-high cells represent presumptive and differentiated proximal tubules, respectively. 
A
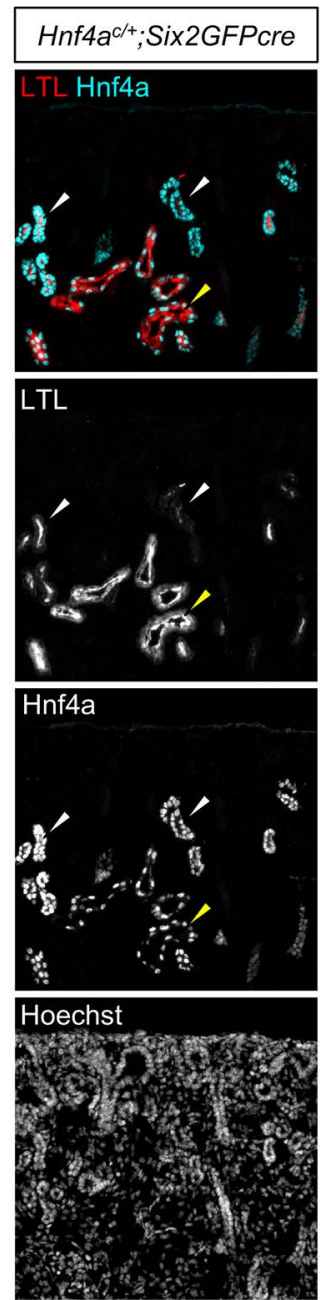
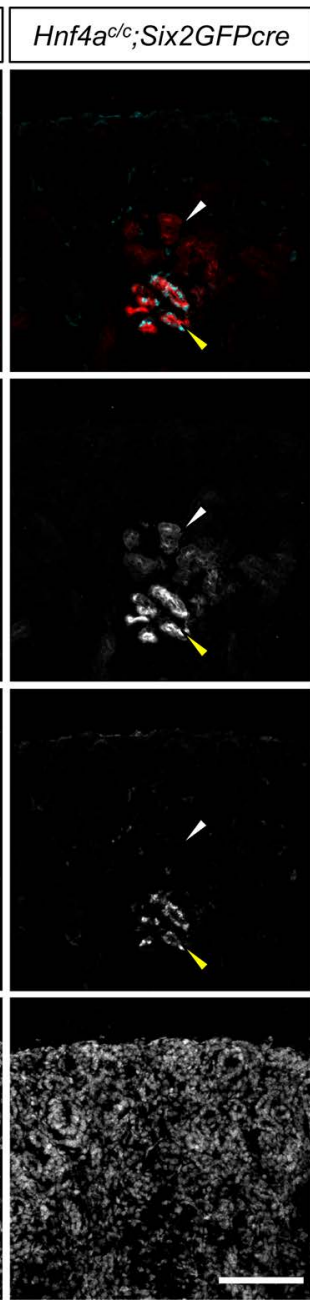

B
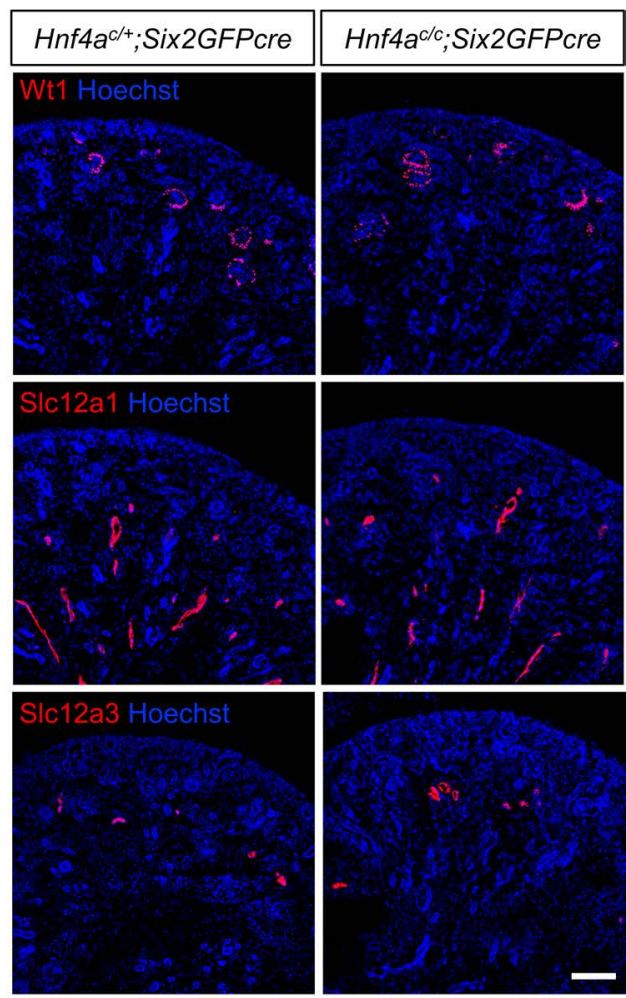

C

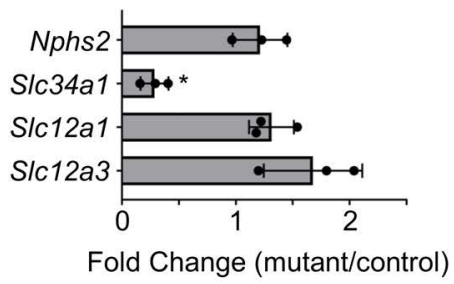

Figure 2. Deletion of Hnf4a by Six2GFPcre leads to a defect in proximal tubule (PT) formation. (A) Loss of Hnf4a in the developing nephron inhibits the formation of differentiated PTs. Presumptive PTs show weak LTL staining at their apical side (marked by white arrowheads, LTL-low). Differentiated PTs have strong LTL staining (marked by yellow arrowheads, LTL-high). The Hnf4a mutant kidney (right column) has fewer LTL-high cells, and these LTL-high cells still express Hnf4a, suggesting that they escaped Cre-mediated deletion of Hnf4a. Images are representative of $n=3$. (B) Deletion of Hnf4a does not affect formation of other nephron segments (WT1, podocytes; SIc12a1, loop of Henle; SIc12a3, distal tubule). Images are representative of $n=4 \mathrm{Hnf} 4 a$ mutants and $n=2$ controls. (C) qPCR analysis of segment-specific markers shows that PT-specific expression of SIc34a1 is significantly lower in the Hnf4a mutant kidney compared with the control (Nphs2, podocyte; SIc34a1, proximal tubule; SIc12a1, loop of Henle; SIc12a3, distal tubule). $n=3$. Error bars indicate \pm SD. ${ }^{*} P<0.05$, determined by 2-tailed Student's $t$ test. Scale bars: $100 \mu \mathrm{m}$.

Loss of Hnfta in the nephron lineage causes a defect in proximal tubule formation. Hnfta-null mice exhibit early embryonic lethality due to gastrulation defects (19). In order to investigate the role of Hnfta during nephrogenesis, we conditionally deleted Hnf4a (20) in the nephron lineage using Six2GFPcre. Hnf4a mutants (Hnf4ac ; Six2GFPcre) were collected at E18.5. Littermate heterozygotes $\left(H n f 4 a^{c /+} ;\right.$ Six2GFPcre) were used as controls. Although there was no apparent difference in kidney size at this stage, Hnf4a mutant kidneys exhibited a defect in proximal tubule formation (Figure 2A).

We found that the Hnf4a mutant kidney had both LTL-high and LTL-low cells. However, the mutant kidney had markedly fewer LTL-high cells than its control (Figure 2A). In the Hnf4a mutant kidney, although LTL-low cells were negative for Hnf4a, LTL-high cells were still positive for Hnf4a, indicating that these cells had escaped Cre-mediated deletion of Hnf4a and still expressed Hnf4a. This result strongly suggests that the Hnf4a mutant nephron progenitors can form LTL-low cells, but these LTL-low cells fail to develop into LTL-high cells. These data are consistent with a model where Hnf4a is required for presumptive proximal tubules (LTL-low cells) to develop into differentiated proximal tubules (LTL-high cells).

As implied by its specific expression in the proximal tubule, we found that the loss of Hnfta did not affect the formation of the other segments of the nephron. We examined nephron segmentation in the 
Hnf4a mutant kidney and found that the formation of podocytes $\left(\mathrm{Wt} 1^{+}\right)$, loops of Henle (Slc12a $\left.1^{+}\right)$, and distal tubules $\left(\mathrm{Slc12}_{2} \mathrm{a}^{+}\right.$) was not affected by deletion of Hnf4a (Figure 2B). In order to quantify nephron segmentation of the Hnf4a mutant kidney, we performed quantitative PCR (qPCR) of genes expressed in specific segments of the nephron (Figure 2C). Our qPCR data showed that proximal tubule-specific expression of Slc34a1 was significantly decreased in the Hnf4a mutant kidney. Although expression of the marker genes for the other nephron segments (Nphs2, podocytes; Slc12a1, loop of Henle; Slc12a3, distal tubule) was slightly upregulated in the Hnf4a mutant kidney, their upregulation was not statistically significant. Taken together, our data demonstrate that loss of Hnf4a specifically inhibits the formation of proximal tubules without affecting the formation of the other segments of the nephron.

Hnf4a is required for the formation of differentiated proximal tubule cells. To determine whether there is differential gene expression in LTL-low cells versus LTL-high cells, we looked at previously published single cell RNA sequencing (scRNA-seq) data of the neonatal mouse kidney (Supplemental Figure 2) (21). scRNAseq analysis identified 2 distinct populations of proximal tubules cells: presumptive proximal tubule and differentiated proximal tubule. While presumptive proximal tubule cells, like differentiated proximal tubule cells, express proximal tubule marker genes, their transcriptional profile is closer to that of epithelial nephron progenitors, as shown by the t-distributed stochastic neighbor embedding (t-SNE) plot of our scRNAseq (Supplemental Figure 2A). We identified Lrp2 as a gene expressed in both presumptive proximal tubule cells and differentiated proximal tubule cells. Lrp2, also known as megalin, encodes an endocytic receptor that is important for uptake of macromolecules and is highly expressed in adult proximal tubules (22). Presumptive proximal tubule cells express Lrp2, as well as low levels of Hnf4a. We also identified Ass1 as a gene that shows high expression in differentiated proximal tubule cells but little expression in presumptive proximal tubule cells. Ass 1 encodes the enzyme argininosuccinate synthase 1, which is a component of the urea cycle (23). Differentiated proximal tubule cells express Lrp2, Hnf4a, and Ass1, whereas presumptive proximal tubule cells express Lrp2 and Hnf4a (Supplemental Figure 2, B-D). There is little expression of Ass1 in presumptive proximal tubule cells (Supplemental Figure 2D). This suggests that Ass1 is a marker for mature, differentiated proximal tubule cells. Based on these data, we hypothesized that LTL-low cells would express Lrp2 but not Ass1 and LTL-high cells would express both Lrp2 and Ass1. We used immunofluorescence staining of Lrp2 and Ass1 to assess their expression in Hnf4a mutant and control kidneys (Figure 3). As predicted by scRNA-seq, in the control kidney, Lrp2 was expressed in LTL-low cells, consistent with the idea that LTL-low cells represent presumptive proximal tubule cells. In the Hnf4a mutant kidney, LTL-low cells were Lrp2 ${ }^{+}$and $\mathrm{Hnf}_{4} \mathrm{a}^{-}$, suggesting that $\mathrm{Hnf} 4 \mathrm{a}$ is not required for Lrp2 expression or the formation of presumptive proximal tubule cells. In the control kidney, Ass 1 was expressed in LTL-high cells with little to no expression in LTL-low cells (Figure 3B), indicating that Ass 1 is a marker of differentiated proximal tubule cells. In the Hnf4a mutant kidney, all Ass $1^{+}$cells were $\mathrm{Hnf}_{4} \mathrm{a}^{+}$, suggesting that these Ass $1^{+}$cells were actually unrecombined "escaper" cells and that Hnf4a is required for Ass 1 expression. This indicates that $H n f 4 a$ is required for the formation of differentiated proximal tubules but is dispensable for presumptive proximal tubule formation.

Loss of Hnf4a causes downregulation of proximal tubule-specific genes. From previously published scRNAseq data (21), we identified genes that are preferentially expressed in each nephron segment. We examined expression of these nephron segmentation marker genes in conventional RNA-seq analysis of Hnf4a mutant and control kidneys at E18.5. Our results revealed that the Hnf4a mutant kidneys showed a significant decrease in the expression of proximal tubule genes, with minimal changes in the expression of other nephron segment genes, reflecting the reduced number of proximal tubule cells (Figure 4A). Gene ontology (GO) analysis (https://david.ncifcrf.gov/)for biological processes showed that the expression of genes involved in metabolism and anion transport was decreased in the Hnf4a mutant kidney (Figure 4B). This is consistent with the fact that the proximal tubule is the major site of organic anion transport in the kidney and has a role in the metabolism of proteins and peptides (24-26). GO analysis for cellular components showed that the expression of genes associated with the brush border membrane, a characteristic of the proximal tubule, was decreased in the Hnf4a mutant kidney (Supplemental Figure 3A). Loss of Hnf4a caused reduced expression of genes associated with abnormal renal absorption and abnormal urine homeostasis in mouse models (Supplemental Figure 3B), also reflecting the paucity of proximal tubule cells in the Hnf4a mutant kidney. These findings are consistent with a role for Hnf4a in proper proximal tubule development.

Hnf4a mutant mice recapitulate an FRTS-like phenotype. To determine whether deletion of Hnf4a leads to characteristics of FRTS in mice, we examined the metabolic phenotype of the Hnf $4 a$ mutant mice. The Hnf4a 
A

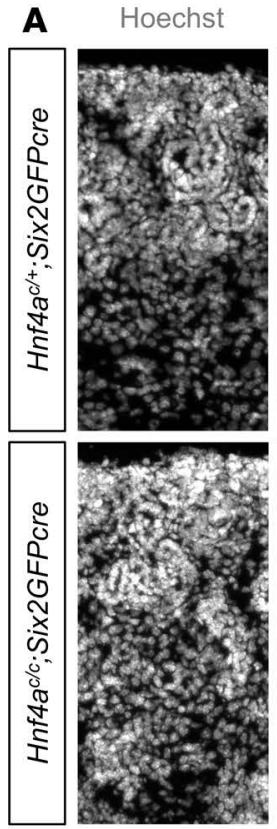

B
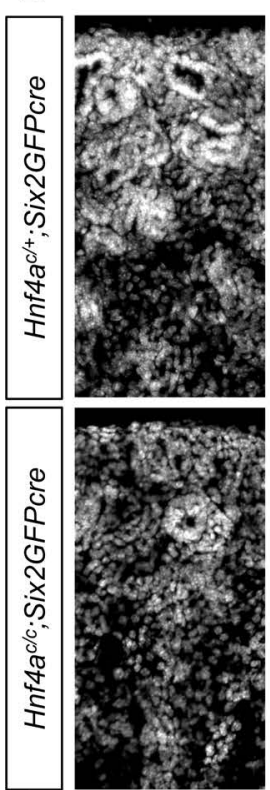

LTL
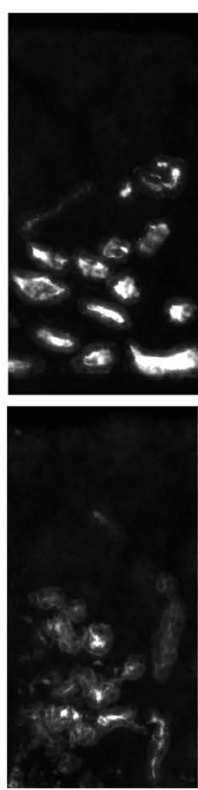

LTL

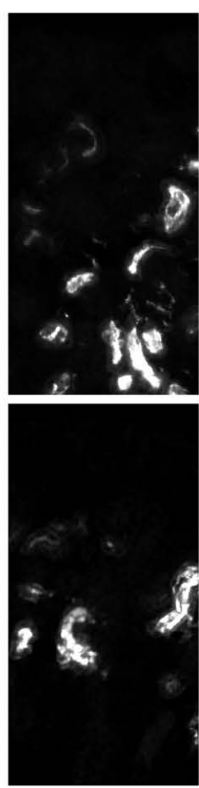

Lrp2
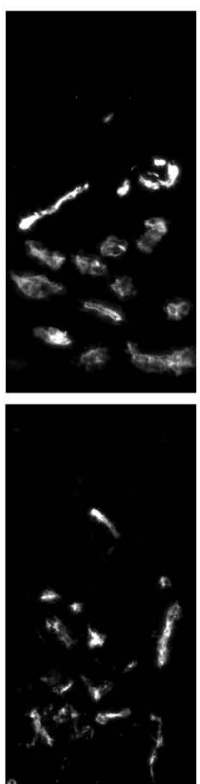

Ass1
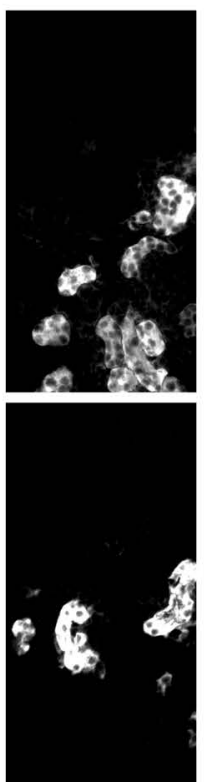

Hnf4a
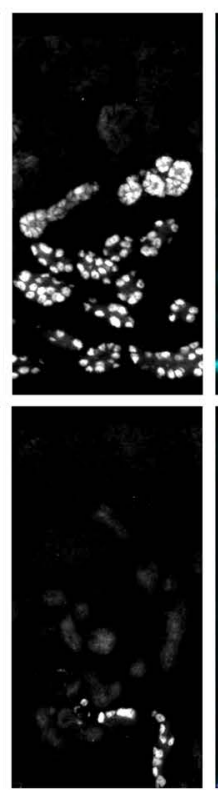

$\mathrm{Hnf4a}$
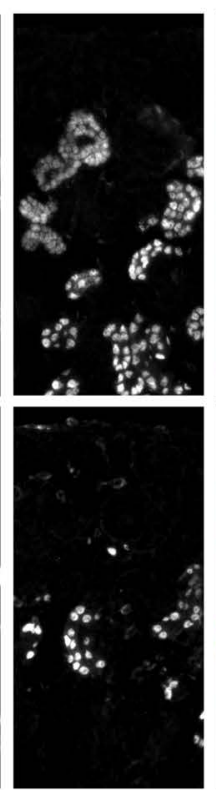

Lrp2 LTL
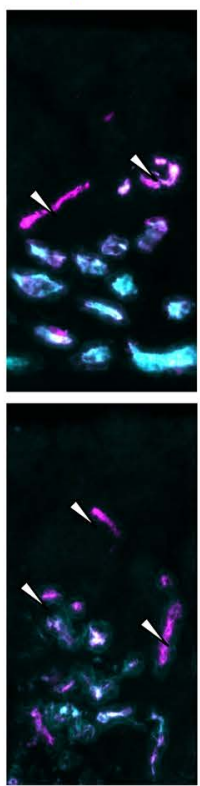

Ass 1 LTL
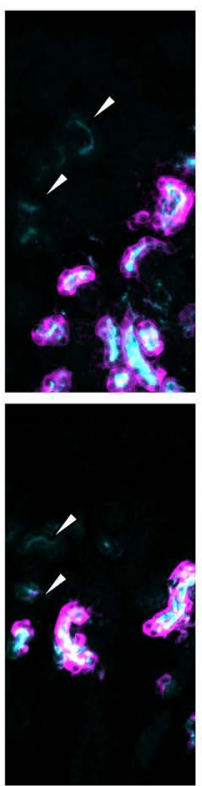

Lrp2 Hnf4a
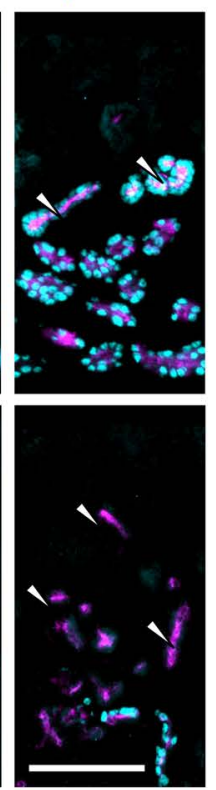

Ass1 Hnf4a
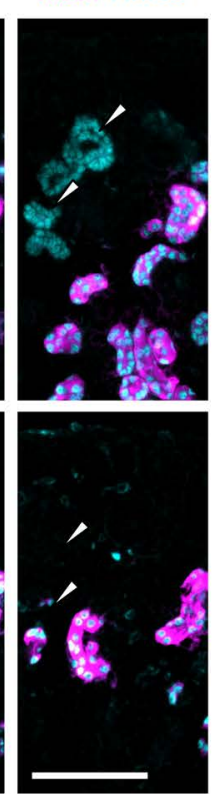

Figure 3. Hnf4a is dispensable for the formation of presumptive PT (LTL-low) cells but required for the formation of differentiated PT (LTLhigh) cells. (A) Lrp2 is detected in LTL-low (white arrowheads) and LTLhigh cells. The Hnf4a mutant kidney has Lrp2 ${ }^{+} \mathrm{Hnf}_{4} \mathrm{a}^{-}$cells, indicating that Hnf4a is dispensable for the formation of presumptive PT cells. (B) Ass1 is detected in LTL-high cells but not in LTL-low cells (white arrowheads) In the Hnf4a mutant kidney, there are no Ass $1^{+} \mathrm{Hnf}_{4} \mathrm{a}^{-}$cells, suggesting that $\mathrm{Hnf} 4 \mathrm{a}$ is required for the formation of differentiated PT cells. Scale bars: $100 \mu \mathrm{m}$. Images are representative of $n=4 H n f 4 a$ mutant and $n=$ 2 control kidneys.

mutant mice were viable, likely due to the nephron progenitors that escaped Cre-mediated deletion of Hnf4a. In order to assess renal function, urine was collected from 2- to 3-month-old Hnf4a mutant mice (Hnf4a ${ }^{c / c}$; Six2GFPcre) and their control littermates ( $H n f 4 a^{c /+}$ or $H n f 4 a^{c /+}$;Six2GFPcre). We measured water intake and urine volume over a period of 24 hours. We found that, similar to FRTS patients, the Hnf4a mutant mice drank more water (polydipsia) and excreted more urine (polyuria) than their control littermates (Figure 5, A and B). Polydipsia can be attributed to polyuria caused by defective reabsorption of water in the proximal tubules (3). To determine the cause of defective water reabsorption, we examined the expression of aquaporins (water transporters) that are known to function in the proximal tubule. Aqp1, Aqp7, and Aqp11 are expressed in the proximal tubules and are important for water reabsorption in the proximal tubule (4, 27, 28). Aqp1-null mice exhibit polydipsia and polyuria, similar to the Hnf4a mutant mice (29). Expression of proximal tubule aquaporins was decreased in the Hnf4a mutant, reflecting the decreased number of proximal tubule cells (Figure 5E). Reduced expression of aquaporin genes in the Hnf4a mutant kidneys most likely causes the polydipsia and polyuria phenotype. 
A

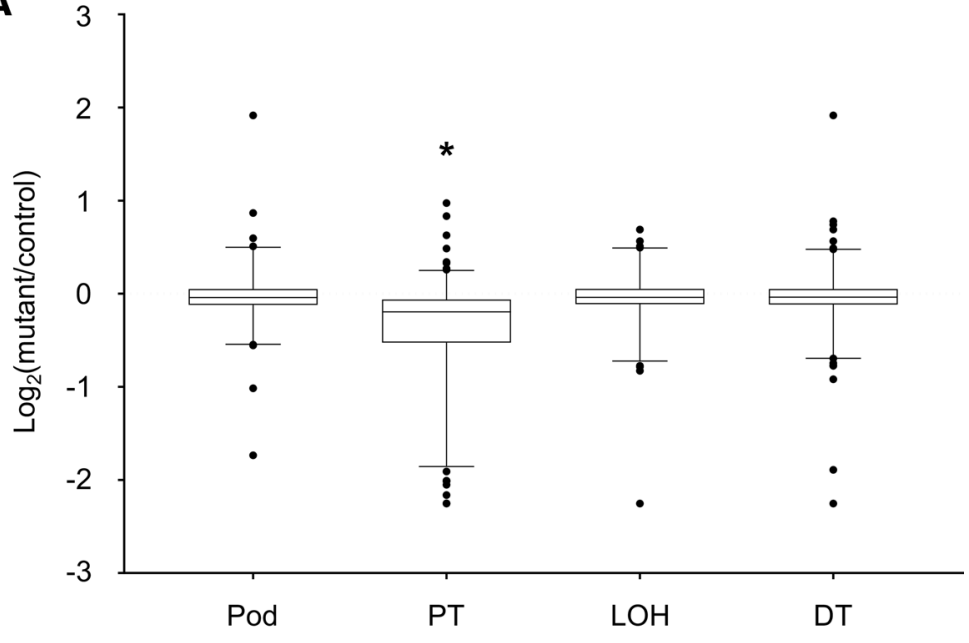

B

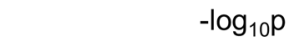

$\begin{array}{llllllllll}0 & 2 & 4 & 6 & 8 & 10 & 12 & 14 & 16 & 18\end{array}$
Figure 4. Deletion of Hnf4a by Six2GFPcre results in decreased expression of PT-specific genes. (A) RNA-seq analysis of E18.5 kidneys shows that loss of $\mathrm{Hnf} 4 \mathrm{a}$ in the nephron lineage primarily affects expression of PT-specific nephron segmentation markers. Results are represented as box plots. The box boundaries represent the upper and lower quartiles, the horizontal line represents the median, the whiskers are drawn to the 1st and 99th percentile, and the dots represent the outlier values. ${ }^{*} P<0.0001$, determined by 1-way ANOVA. Pod, podocyte; PT, proximal tubule; LOH, loop of Henle; DT, distal tubule. (B) Gene ontology analysis with PT-specific genes that are downregulated in the Hnf4a mutant kidney at E18.5 shows enrichment of genes associated with metabolism and transport.

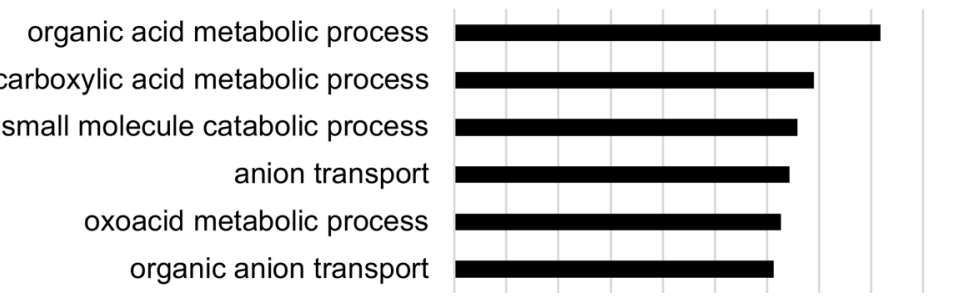

Urinalysis showed that the Hnf4a mutant mice excreted more glucose (glycosuria) and phosphate (phosphaturia) into their urine compared with control littermates (Figure 5, C and D). Since proximal tubule cells are responsible for reabsorption of glucose and phosphate, excess amounts of these solutes in the urine indicate proximal tubule dysfunction $(2,3)$. The principal glucose transporters expressed in the proximal tubule are Slc5a1, Slc5a2, and Slc2a2 (30). Slc5a1 and Slc5a2 are sodium-dependent glucose transporters (SGLTs) responsible for glucose reabsorption in the proximal tubule $(30,31)$. Mutations in $S L C 5 A 1$ and $S L C 5 A 2$ are associated with glucose-galactose malabsoprtion and familial renal glycosuria, respectively (31). Slc2a2 is a facilitated glucose transporter (GLUT2) that is responsible for the transport of glucose through the basolateral membrane of proximal tubule cells $(31,32)$. Slc2a2 $\mathrm{KO}$ in mice induces glycosuria (33). SLC2A2 deficiency is linked to Fanconi-Bickel syndrome, a glycogen storage disease (31). Many of these patients also present with glycosuria $(32,34)$. Since the $H n f 4 a$ mutants presented with renal glycosuria (Figure 5C), it is likely that they have a loss of glucose transporters. RNA-seq analysis showed that the expression of proximal tubule glucose transporters was decreased in the Hnf4a mutant mice (Figure 5E), implicating loss of glucose transporters as the cause of the renal glycosuria in the Hnf4a mutant mice.

The principal sodium-phosphate transporters expressed in the proximal tubule are Slc20a2, Slc34a1, and $\operatorname{Slc34a3}(35,36)$. These phosphate transporters are located in the apical brush border membrane of the proximal tubule, and loss of these transporters leads to phosphaturia (35). RNA-seq analysis confirmed decreased expression of the proximal tubule phosphate transporters in the Hnf4a mutant (Figure 5E). This is consistent with the increased excretion of urinary phosphate in the Hnf4a mutant mice (Figure 5D). The amount of phosphate reabsorbed by the proximal tubules is dependent on the number of phosphate transporters (37), suggesting the loss of the proximal tubule cells in the Hnf4a mutant mice caused loss of phosphate transporters, leading to the phosphaturia phenotype. These data suggest that loss of transporters due to paucity of proximal tubule cells contributes to the FRTS-like phenotype in the Hnf4a mutant mice.

Hnf4a mutant mice display renal tubular dysgenesis and nephrocalcinosis. In order to investigate the renal pathology of the FRTS phenotype, we examined the morphology of the Hnfta mutant kidney. The kidneys of adult Hnf4a mutant mice were smaller than their controls (data not shown). The Hnf4a mutant kidney 
A
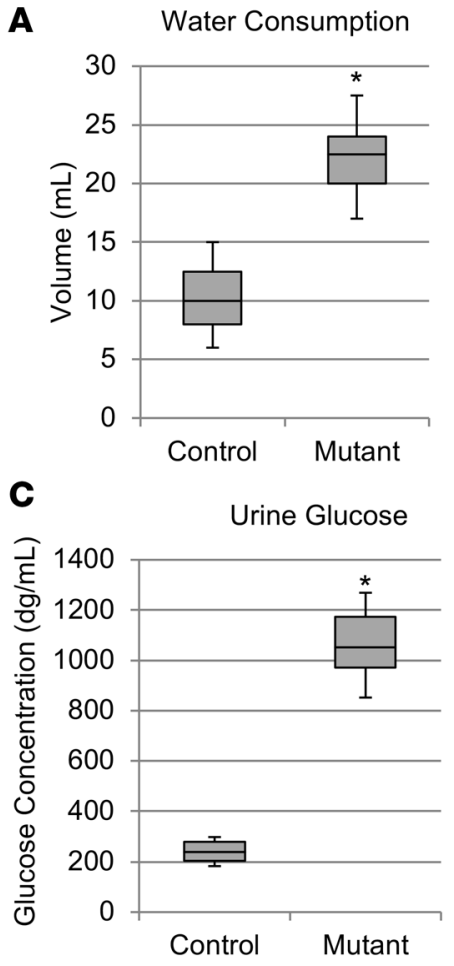

B

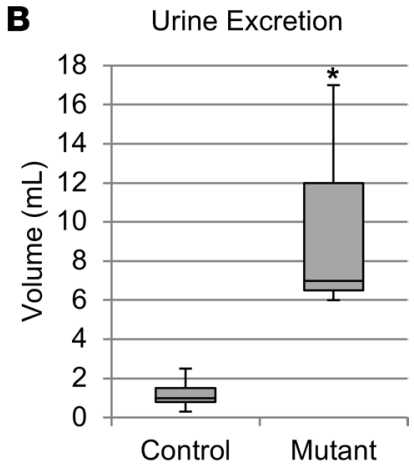

D

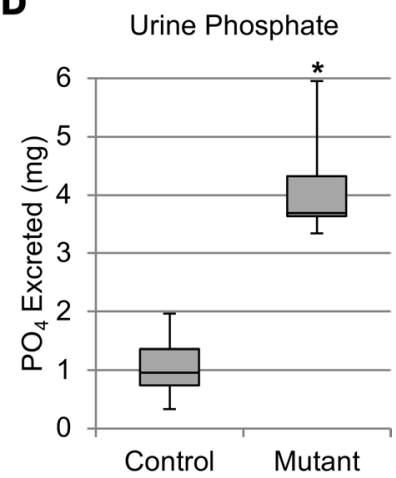

Figure 5. Hnf4a mutant mice recapitulate FRTS phenotypes. Symptoms include polydipsia (A), polyuria (B), increased urine glucose concentration (C), and excessive phosphate excretion (D). Water and urine were measured for 24 hours. Results are represented as box plots. The box boundaries represent the upper and lower quartiles, the horizontal line represents the median, and the whiskers represent the minimum and maximum values. Control, $\mathrm{Hnf} 4 \mathrm{a}^{c /+}$;Six2GFPcre or $\mathrm{Hnf} 4 \mathrm{a}^{c /+}$. Mutant, Hnf4a $a^{c / c}$;Six2GFPcre. $n=$ $9 ;{ }^{*} P<0.05$, determined by 2 -tailed Student's $t$ test. (E) RNAseq analysis of Hnf4a mutant kidneys at E18.5 shows decreased expression of genes encoding phosphate, glucose, and water transporters, consistent with the PT dysfunction phenotype. $n=2$.

E Water
Transporters

Glucose Transporters

Phosphate Transporters

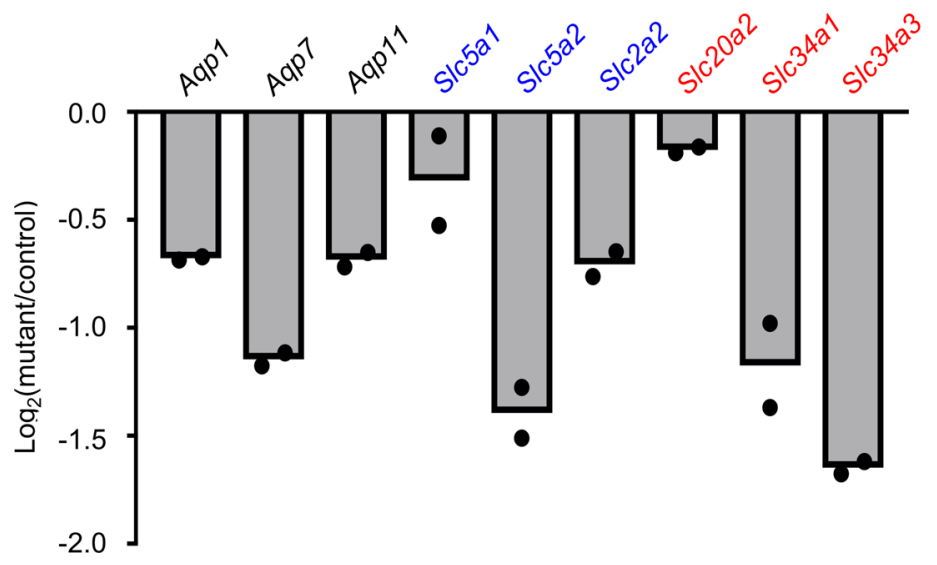

was highly disorganized with fewer proximal tubules (Figure 6A). All of the proximal tubule cells in the $H n f 4 a$ mutant kidney were positive for $\mathrm{Hnf} 4 \mathrm{a}$, indicating that these $\mathrm{Hnf4a}^{+}$cells had escaped Cre-mediated deletion of Hnf4a. Patients with the R76W mutation in HNF4A are known to present with nephrocalcinosis (7). Nephrocalcinosis refers to the deposition of calcium in the renal parenchyma. Loss of the phosphate transporter Slc34a1 has also been linked to nephrocalcinosis in mouse models (38). The Hnf4a mutant mice had decreased expression of Slc34a1 (Figure 2C and Figure 5E), suggesting that the Hnf4a mutant mice may have nephrocalcinosis. In order to examine calcium deposition in the mutant kidney, we performed von Kossa staining on paraffin sections of 2-month-old adult kidneys. Hnf4a mutant kidneys showed calcium accumulation in the renal tubules, suggesting that the loss of Hnf4a causes nephrocalcinosis (Figure 6B). This reaffirms that the Hnf4a mutant mice recapitulate the FRTS patient phenotype.

\section{Discussion}

The nephron is segmented into the renal corpuscle, proximal tubule, loop of Henle, and distal tubule along the proximal-distal axis. Different nephron tubule segments contain specific types of epithelial cells that carry out distinct physiological functions and collectively act as a blood filtration unit. It is still poorly understood how 


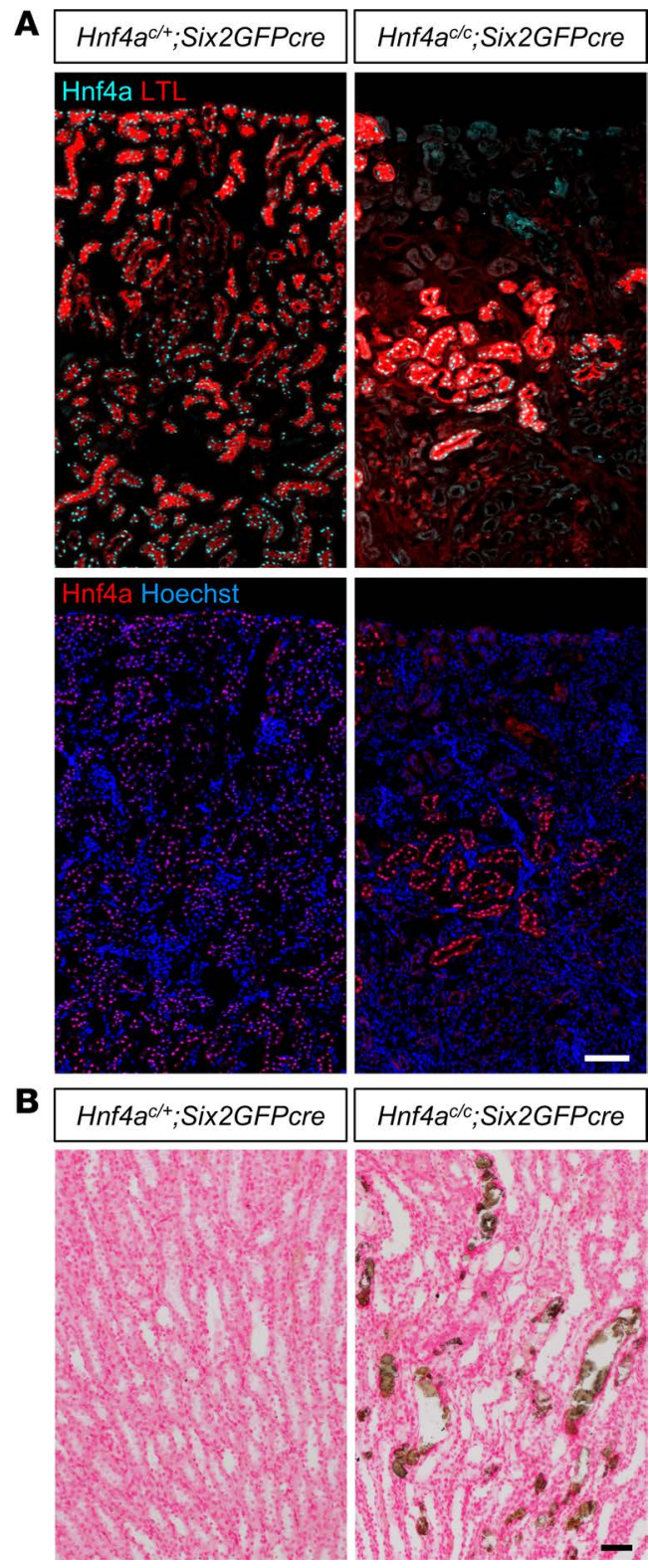

Figure 6. Adult Hnf4a mutant has disorganized PTs and nephrocalcinosis. (A) Representative immunofluorescence staining of 2-month-old mouse kidney $(n=3)$. Mutant kidney has fewer LTL stain-positive PTs. (B) Representative von Kossa staining of 2-month-old mouse kidney $(n=3)$. Mutant kidney has calcium deposits in renal tubules. Scale bars: $100 \mu \mathrm{m}$.

distinct segmental identities of the nephron are specified during nephrogenesis. Here, we show that Hnf4a is a key transcription factor that controls the development of the proximal tubule segment.

Proximal tubules are the most abundant cell type present in the nephron. Its major function is reabsorption. Defective proximal tubule function causes FRTS, which includes symptoms such as impaired reabsorption of phosphate, amino acids, glucose, and other organic solutes (1). Currently, treatment for FRTS includes replacement of the lost solutes by administering fluids and electrolytes $(1,3)$. Even with treatment, FRTS can lead to chronic kidney failure in adolescence or adulthood (1). Previous mouse models of FRTS have utilized either KO of endocytic genes, such as megalin and cubilin, or the administration of heavy metals to induce FRTS (39). Our mouse model utilizes a gene that has been linked to inherited FRTS in human patients. A heterozygous R76W mutation in the HNF4A gene was identified in 3 family members with FRTS (7). This mutation was subsequently identified in 3 unrelated carriers with FRTS (7). From our findings, we can speculate that the HNF4A R76W mutation is a loss-of-function or hypomorphic mutation, likely causing paucity of proximal tubules. Since the R76 residue is located in the DNA-binding domain of the $H N F 4 A$ gene (40), it is likely that the R76W mutation interferes with the binding of Hnf4a to its target genes.

Considering the important roles of proximal tubules in renal function, a complete lack of proximal tubules would cause lethality. However, the mosaic expression of Six2GFPcre in our mouse model causes a subset of nephron progenitor cells to escape Cre-mediated deletion of Hnfta in the mutant kidney, allowing most of the mutant mice to survive with substantially reduced numbers of proximal tubules. The paucity of proximal tubules caused the mutant mice to recapitulate FRTS phenotypes. In addition to polyuria, polydipsia, glycosuria, and phosphaturia, the Hnf4a mutant mice exhibited nephrocalcinosis, highlighting the importance of proximal tubule function in renal regulation of calcium.

Our study shows that proximal tubule development is a multistep process. By analyzing previously published scRNA-seq of the newborn mouse kidney (21), we identified 2 cell populations that represent different stages of proximal tubule development. Both presumptive proximal tubules and differentiated proximal tubules express Lrp2 and Hnf4a, 2 well-known proximal tubule marker genes. However, we observed that the expression of other proximal tubule marker genes, such as Slc34a1 and Ass1, is largely absent in presumptive proximal tubules, indicating their immature status. It appears that expression of these genes is activated after differentiated proximal tubules are formed. Our data strongly suggest that Hnf4a regulates the development of presumptive proximal tubules into differentiated proximal tubules.

Further investigation is required to characterize other regulators of proximal tubule development. The previous model of proximal tubule development suggested that Notch signaling promotes the formation of the proximal tubule and represses the formation of the distal tubule segment (41-43). However, we have recently shown that Notch signaling is required for the formation of all nephron segments, and activation of Notch signaling in the developing nephron shows no effect on nephron segmentation in the mouse kidney $(44,45)$. It has been shown that, in the zebrafish pronephros, retinoic acid signaling promotes the formation of proximal tubules and represses the formation of distal tubules $(46,47)$. Considering the clear role of retinoic acid signaling in pronephros segmentation, it will be interesting to investigate potential interaction between retinoic acid signaling and Hnf4a in mammalian proximal tubule segmentation. It is possible that retinoic acid signaling may regulate proximal tubule development by activating the expression of Hnf4a; alternatively, Hnf4a and retinoic acid receptors may coordinate to promote the development of proximal tubules by regulating common target genes, as shown in human hepatocytes $(10,48,49)$. 


\section{Methods}

Mice. All mouse alleles used in this study have been previously published: Six $2^{\text {tml(tTA,tetO-EGFP/cre)Amc }}$ (Six2GFPcre) $(14,15) ; H n f 4 a^{\text {tm ISad }}\left(H n f 4 a^{c / c}\right)(20)$; and Gt(ROSA)26Sor ${ }^{\text {tm3(CAG-EYFP)Hze }}\left(\right.$ Rosa26 $\left.^{\text {EYFP }}\right)$ (16).

Immunofluorescence. Embryonic, neonatal, and adult murine kidneys were fixed in 4\% PFA/PBS, incubated overnight in $10 \%$ sucrose/PBS at $4^{\circ} \mathrm{C}$, and imbedded in OCT (Thermo Fisher Scientific). Cryosections $(9 \mu \mathrm{m})$ were incubated overnight with primary antibodies in $5 \%$ heat-inactivated sheep serum/PBST (PBS [Corning] with $0.1 \%$ Triton X-100 [AMRESCO]). We used the primary antibodies for GFP (1:500, Aves, GFP-1020), Jag1 (1:20, Developmental Studies Hybridoma Bank [DSHB], TS1.15H), Wt1 (1:100, Santa Cruz Biotechnology Inc., sc-7385), Biotin-LTL (1:500, Vector Laboratories, B-1325), FITC-LTL (1:200, Vector Laboratories, FL-1321), Slc12a1 (1:500, Proteintech, 18970-1-AP), Slc12a3 (1:300, MilliporeSigma, HPA028748), Hnf4a (1:1,000, Santa Cruz Biotechnology Inc., sc-8987X; or 1:500, Abcam, ab41898), Lrp2 (1:100, Santa Cruz Biotechnology Inc., sc-515772), and Ass1 (1:500, Proteintech, 162101-AP). Fluorophore-labeled secondary antibodies were used for indirect visualization of the target. Images were taken with a Nikon Ti-E widefield microscope equipped with an Andor Zyla camera and Lumencor SpectraX light source housed at the Confocal Imaging Core (CIC) at CCHMC.

$q P C R$. Control or Hnf4a mutant embryonic kidneys at E18.5 were placed in RNAlater (Invitrogen) overnight at $4^{\circ} \mathrm{C}$. Total RNA was extracted using the Qiagen RNeasy Micro Kit (Qiagen 74004) according to manufacturer's instructions for dissected animal tissues. RNA concentration was measured with NanoDrop 2000c (Thermo Fisher Scientific). Using about $2 \mu \mathrm{g}$ total RNA, reverse transcription was performed using the RevertAid cDNA Synthesis Kit (Thermo Fisher Scientific, K1621). qPCR was performed on an Applied Biosystems StepOne Plus (Thermo Fisher Scientific) using Power SYBR Green PCR Master Mix (Thermo Fisher Scientific, 4368706). Oligonucleotide primers (5'-3', forward and reverse) used were: Gapdh, CAACTTTGTCAAGCTCATTTCCTG and CCTCTCTTGCTCAGTGTCCTT; Nphs2, CTCTGGCCCTAACATCTCCA and TTCAGTGAGCAAGCAACCAG; Slc34a1, TGCTGAGAGACACTCCGTTG and TATTGGGGTGGCAAATTCTC; Slc12a1, AGCGGGCTCTCCTTAAGTTC and CTCAGGAGGCCAAGCAGAAT; and Slc12a3, AGCTGGAGAAGAGGCTTCAA and TGCAACTTCAAGGTCCAGAA. Two biological replicates of control kidneys and 3 biological replicates of Hnf4a mutant kidneys were used. Fold expression calculations were obtained using the $\Delta \Delta \mathrm{Ct}$ method.

von Kossa staining. Adult murine kidneys were fixed in $4 \%$ PFA/PBS overnight, dehydrated in $50 \%$ ethanol/PBS and $70 \%$ ethanol/PBS, and stored at $4{ }^{\circ} \mathrm{C}$ in $70 \%$ ethanol/PBS before washing in xylene and embedding in paraffin (Thermo Fisher Scientific). von Kossa staining was performed on paraffin sections (5 $\mu \mathrm{m})$ by the CCHMC Pathology Research Core using standard protocols. Stained slides were imaged using a Nikon 90 i upright widefield microscope.

$R N A$-seq. We isolated mRNA from $1 \mu \mathrm{g}$ total RNA using NEBNext Poly(A) mRNA Magnetic Isolation Module (New England Biolabs [NEB], E7490) following manufacturer's instructions. Fragmentation of RNA followed by reverse transcription and second strand cDNA synthesis was done using NEBNext Ultra RNA Library Prep Kit for Illumina (NEB, E7530) following manufacturer's instructions. The resulting double-stranded cDNAs were further processed to DNA sequencing libraries using ThruPLEX DNA-seq 12S Kit (Clontech Laboratories, R400428). Libraries were size-selected by gel purification for an average size of $350 \mathrm{bp}$. Each purified library was quantified using a Qubit fluorometer (Invitrogen), and equal amounts of each library were pooled and submitted for sequencing on the Illumina HiSeq 2500 by the DNA Sequencing and Genotyping Core at CCHMC. All RNA-seq reads were aligned to mm9 using TopHat (ver2.0.11), and the BAM files were generated using Samtools (ver0.1.19). Normalized gene expression values were calculated using Cufflinks (ver2.1.1). Data are available in Gene Expression Omnibus under accession number GSE112828 (https://www.ncbi.nlm.nih.gov/geo/query/acc.cgi?acc=GSE112828).

GO analysis. GO analysis was performed using DAVID Bioinformatics Resources on differentially expressed genes identified from the RNA-seq analysis (50, 51).

scRNA-seq data analysis. scRNA-seq (drop-seq) analysis of P1 mouse kidney cells (GSE94333) was previously described (21). The cells from 4 drop-seq experiments were merged, and batch effects were minimized using Seurat's canonical correlation analysis. Cell-type clusters and marker genes were identified using the R3.4.1 library Seurat2.0.1. All clustering was unsupervised, without driver genes. The influence of the number of unique molecular identifiers was minimized using Seurat's RegressOut function. Initial cell filtering selected cells that expressed >1,000 genes. Genes included in the analysis were expressed in a minimum of 3 cells. Only 1 read per cell was needed for a gene to be counted as expressed 
per cell. The resulting gene expression matrix was normalized to 10,000 molecules per cell and log transformed. Cells containing high percentages of mitochondrial, histone, or hemoglobin genes were filtered out. Genes with the highest variability among cells were used for principal components analysis. Clustering was performed with Seurat's t-SNE implementation using significant principal components determined by JackStraw plot. Marker genes were determined for each cluster using Seurat's FindAllMarkers function using genes expressed in a minimum of $10 \%$ of cells and fold change threshold of 1.3 . Over/under-clustering was verified via gene expression heatmaps.

Urine analysis. Nine Hnf4a mutant mice and their control littermates ( 5 males and 4 females) were placed individually in metabolic cages (Tecniplast, 01-814-25, Thermo Fisher Scientific). Mice were fed and received water ad libitum. Food and water consumption was measured for 24 hours. Urine was collected after 24 hours. Urine samples were centrifuged at $1,690 \mathrm{~g}$ for 5 minutes at $4^{\circ} \mathrm{C}$. The supernatant was stored at $-80^{\circ} \mathrm{C}$ until testing. Glucose concentration tests and phosphate concentration tests were performed by Cincinnati Veterinary Laboratory Inc. and IDEXX Laboratories.

Statistics. Box-and-whiskers plots show median (line in box), 25th and 75th percentile (lower and upper edges of box, respectively), and minimum and maximum values (whiskers). For the RNA-seq data, the whiskers are drawn to the 1st percentile and the 99th percentile. Comparisons between RNA-seq analysis of nephron segmentation markers were assessed using a 1-way ANOVA with Tukey's HSD post-hoc analysis. Two-tailed Student's $t$ test was used for comparisons of qPCR analysis between the control and mutant. Two-tailed Student's $t$ test was used for comparison of urinalysis results between the control and mutant groups. $P<0.05$ was considered to be statistically significant.

Study approval. All experiments were performed in accordance with animal care guidelines, and the protocol was approved by the IACUC of the CCHMC (IACUC2017-0037 and IACUC2017-0011). We adhere to the NIH Guide for the Care and Use of Laboratory Animals (National Academies Press, 2011).

\section{Author contributions}

SSM performed experiments. EC performed conventional RNA-seq. MA and SSP performed scRNA-seq analysis. SSM and JSP designed the experiments, analyzed the data, and cowrote the manuscript.

\section{Acknowledgments}

We thank Matt Kofron and the CIC at CCHMC for help with microscopy and Elizabeth Mann for help with urinalysis. This work was supported by the NIH/NIDDK (R01 DK100315 to JSP) and the National Institutes of Health/NHLBI (T32 HL007752 to SSM).

Address correspondence to: Joo-Seop Park, Cincinnati Children's Hospital Medical Center, R1566, ML7007, 3333 Burnet Avenue, Cincinnati, Ohio 45229, USA. Phone: 513.803.7871; Email: joo-seop.park@cchmc.org.

1. Litzinger MHJ, Boatman MD, Talatala E, Litzinger M. Fanconi syndrome. US Pharm. 2011;36(6):HS12-HS16.

2. Walsh SB, Unwin RJ. Renal tubular disorders. Clin Med (Lond). 2012;12(5):476-479.

3. Igarashi T. Pediatric Fanconi syndrome. In: Avner ED, Harmon WE, Niaudet P, Yoshikawa N, Emma F, Goldstein SL, eds. Pediatric Nephrology. Berlin, Germany: Springer-Verlag Berlin and Heidelberg GmbH \& Co. KG; 2016:1355-1388

4. Baum M, Quigley R. Proximal tubule water transport-lessons from aquaporin knockout mice. Am J Physiol Renal Physiol. 2005;289(6):F1193-F1194.

5. Klootwijk ED, Reichold M, Unwin RJ, Kleta R, Warth R, Bockenhauer D. Renal Fanconi syndrome: taking a proximal look at the nephron. Nephrol Dial Transplant. 2015;30(9):1456-1460.

6. Blaine J, Chonchol M, Levi M. Renal control of calcium, phosphate, and magnesium homeostasis. Clin J Am Soc Nephrol. 2015;10(7):1257-1272.

7. Hamilton AJ, et al. The HNF4A R76W mutation causes atypical dominant Fanconi syndrome in addition to a $\beta$ cell phenotype. J Med Genet. 2014;51(3):165-169.

8. Sladek FM, Zhong WM, Lai E, Darnell JE. Liver-enriched transcription factor HNF-4 is a novel member of the steroid hormone receptor superfamily. Genes Dev. 1990;4(12B):2353-2365.

9. Hayhurst GP, Lee YH, Lambert G, Ward JM, Gonzalez FJ. Hepatocyte nuclear factor 4alpha (nuclear receptor 2A1) is essentia for maintenance of hepatic gene expression and lipid homeostasis. Mol Cell Biol. 2001;21(4):1393-1403.

10. Hatzis P, Talianidis I. Regulatory mechanisms controlling human hepatocyte nuclear factor 4alpha gene expression. Mol Cell Biol. 2001;21(21):7320-7330

11. Thiagarajan $\mathrm{RD}$, et al. Identification of anchor genes during kidney development defines ontological relationships, molecular subcompartments and regulatory pathways. PLoS One. 2011;6(2):e17286.

12. Lee JW, Chou CL, Knepper MA. Deep Sequencing in Microdissected Renal Tubules Identifies Nephron Segment-Specific 
Transcriptomes. J Am Soc Nephrol. 2015;26(11):2669-2677.

13. Martovetsky G, Tee JB, Nigam SK. Hepatocyte nuclear factors $4 \alpha$ and $1 \alpha$ regulate kidney developmental expression of drug-metabolizing enzymes and drug transporters. Mol Pharmacol. 2013;84(6):808-823.

14. Kobayashi A, et al. Six2 defines and regulates a multipotent self-renewing nephron progenitor population throughout mammalian kidney development. Cell Stem Cell. 2008;3(2):169-181.

15. Park JS, Valerius MT, McMahon AP. Wnt/beta-catenin signaling regulates nephron induction during mouse kidney development. Development. 2007;134(13):2533-2539.

16. Madisen L, et al. A robust and high-throughput Cre reporting and characterization system for the whole mouse brain. Nat Neurosci. 2010;13(1):133-140.

17. Schulte BA, Spicer SS. Histochemical evaluation of mouse and rat kidneys with lectin-horseradish peroxidase conjugates. Am $J$ Anat. 1983;168(3):345-362.

18. Hennigar RA, Schulte BA, Spicer SS. Heterogeneous distribution of glycoconjugates in human kidney tubules. Anat Rec. $1985 ; 211(4): 376-390$.

19. Chen WS, et al. Disruption of the HNF-4 gene, expressed in visceral endoderm, leads to cell death in embryonic ectoderm and impaired gastrulation of mouse embryos. Genes Dev. 1994;8(20):2466-2477.

20. Parviz F, Li J, Kaestner KH, Duncan SA. Generation of a conditionally null allele of hnf4alpha. Genesis. 2002;32(2):130-133.

21. Adam M, Potter AS, Potter SS. Psychrophilic proteases dramatically reduce single-cell RNA-seq artifacts: a molecular atlas of kidney development. Development. 2017;144(19):3625-3632.

22. Leheste JR, et al. Megalin knockout mice as an animal model of low molecular weight proteinuria. Am J Pathol. 1999;155(4):1361-1370.

23. Engel K, Höhne W, Häberle J. Mutations and polymorphisms in the human argininosuccinate synthetase (ASS1) gene. Hum Mutat. 2009;30(3):300-307.

24. Dantzler WH. Renal organic anion transport: a comparative and cellular perspective. Biochim Biophys Acta. 2002;1566(1-2):169-181.

25. Nieskens TT, et al. A Human Renal Proximal Tubule Cell Line with Stable Organic Anion Transporter 1 and 3 Expression Predictive for Antiviral-Induced Toxicity. AAPS J. 2016;18(2):465-475.

26. Carone FA, Peterson DR, Oparil S, Pullman TN. Renal tubular transport and catabolism of proteins and peptides. Kidney Int. 1979;16(3):271-278.

27. Nielsen S, Frøkiaer J, Marples D, Kwon TH, Agre P, Knepper MA. Aquaporins in the kidney: from molecules to medicine. Physiol Rev. 2002;82(1):205-244.

28. Agarwal SK, Gupta A. Aquaporins: The renal water channels. Indian J Nephrol. 2008;18(3):95-100.

29. Schnermann J, Chou CL, Ma T, Traynor T, Knepper MA, Verkman AS. Defective proximal tubular fluid reabsorption in transgenic aquaporin-1 null mice. Proc Natl Acad Sci USA. 1998;95(16):9660-9664.

30. Triplitt CL. Understanding the kidneys' role in blood glucose regulation. Am J Manag Care. 2012;18(1 Suppl):S11-S16.

31. Szablewski L. Distribution of glucose transporters in renal diseases. J Biomed Sci. 2017;24(1):64.

32. Mueckler M, Thorens B. The SLC2 (GLUT) family of membrane transporters. Mol Aspects Med. 2013;34(2-3):121-138

33. Guillam MT, et al. Early diabetes and abnormal postnatal pancreatic islet development in mice lacking Glut-2. Nat Genet. 1997;17(3):327-330.

34. Santer R, Schneppenheim R, Dombrowski A, Götze H, Steinmann B, Schaub J. Mutations in GLUT2, the gene for the liver-type glucose transporter, in patients with Fanconi-Bickel syndrome. Nat Genet. 1997;17(3):324-326.

35. Blaine J, Weinman EJ, Cunningham R. The regulation of renal phosphate transport. Adv Chronic Kidney Dis. 2011;18(2):77-84

36. Lederer E. Renal phosphate transporters. Curr Opin Nephrol Hypertens. 2014;23(5):502-506.

37. Forster IC, Hernando N, Biber J, Murer H. Proximal tubular handling of phosphate: A molecular perspective. Kidney Int. 2006;70(9):1548-1559.

38. Chau H, El-Maadawy S, McKee MD, Tenenhouse HS. Renal calcification in mice homozygous for the disrupted type IIa Na/Pi cotransporter gene Npt2. J Bone Miner Res. 2003;18(4):644-657.

39. Sirac C, Bridoux F, Essig M, Devuyst O, Touchard G, Cogné M. Toward understanding renal Fanconi syndrome: step by step advances through experimental models. Contrib Nephrol. 2011;169:247-261.

40. Chandra V, Huang P, Potluri N, Wu D, Kim Y, Rastinejad F. Multidomain integration in the structure of the HNF-4 $\alpha$ nuclear receptor complex. Nature. 2013;495(7441):394-398.

41. Cheng HT, Miner JH, Lin M, Tansey MG, Roth K, Kopan R. Gamma-secretase activity is dispensable for mesenchyme-to-epithelium transition but required for podocyte and proximal tubule formation in developing mouse kidney. Development. 2003;130(20):5031-5042.

42. Cheng HT, Kopan R. The role of Notch signaling in specification of podocyte and proximal tubules within the developing mouse kidney. Kidney Int. 2005;68(5):1951-1952.

43. Cheng HT, et al. Notch2, but not Notch1, is required for proximal fate acquisition in the mammalian nephron. Development. 2007;134(4):801-811.

44. Chung E, Deacon P, Marable S, Shin J, Park JS. Notch signaling promotes nephrogenesis by downregulating Six2. Development. 2016;143(21):3907-3913.

45. Chung E, Deacon P, Park JS. Notch is required for the formation of all nephron segments and primes nephron progenitors for differentiation. Development. 2017;144(24):4530-4539.

46. Wingert RA, et al. The cdx genes and retinoic acid control the positioning and segmentation of the zebrafish pronephros. PLoS Genet. 2007;3(10):1922-1938.

47. Li Y, Cheng CN, Verdun VA, Wingert RA. Zebrafish nephrogenesis is regulated by interactions between retinoic acid, mecom, and Notch signaling. Dev Biol. 2014;386(1):111-122.

48. Qian A, Cai Y, Magee TR, Wan YJ. Identification of retinoic acid-responsive elements on the HNF1alpha and HNF4alpha genes. Biochem Biophys Res Commun. 2000;276(3):837-842.

49. Zolfaghari R, Ross AC. Hepatocyte nuclear factor $4 \alpha(\mathrm{HNF} 4 \alpha)$ in coordination with retinoic acid receptors increases all-trans-retinoic acid-dependent CYP26A1 gene expression in HepG2 human hepatocytes. J Cell Biochem. 2014;115(10):1740-1751. 
50. Huang da W, Sherman BT, Lempicki RA. Systematic and integrative analysis of large gene lists using DAVID bioinformatics resources. Nat Protoc. 2009;4(1):44-57.

51. Huang da W, Sherman BT, Lempicki RA. Bioinformatics enrichment tools: paths toward the comprehensive functional analysis of large gene lists. Nucleic Acids Res. 2009;37(1):1-13. 